\title{
Test Results on Heavily Irradiated Silicon Detectors for the CMS Experiment at LHC
}

C. Bozzi ${ }^{13, *}$, S. Albergo ${ }^{5}$, M.M. Angarano ${ }^{2}$, P. Azzi ${ }^{11}$, E. Babucci ${ }^{12}$, N. Bacchetta ${ }^{11}$, A. Bader ${ }^{2}$, G. Bagliesi ${ }^{13}$, P. Bartalini ${ }^{12}$, A. Basti ${ }^{13}$, U. Biggeri ${ }^{7}$, G.M. Bilei ${ }^{12}$, D. Bisello ${ }^{11}$, D. Boemi ${ }^{5}$, F. Bosi ${ }^{13}$, L. Borrello ${ }^{13}$, S. Braibant ${ }^{6}$, H. Breuker ${ }^{6}$, M. Bruzzi ${ }^{7}$, A. Candelori ${ }^{11}$, A. Caner $^{6}$, R. Castaldi ${ }^{13}$, A. Castro $^{11}$, E. Catacchini ${ }^{7}$, B. Checcucci ${ }^{12}$, P. Ciampolini ${ }^{12}$, C. Civinini ${ }^{7}$, D. Creanza ${ }^{2}$, R. D' Alessandro ${ }^{7}$, M. Da

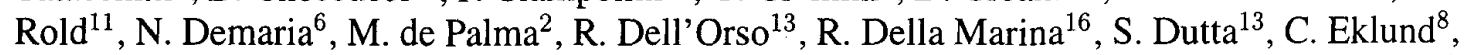
A. Elliott-Peisert ${ }^{6}$, L. Feld ${ }^{6}$, L. Fiore ${ }^{2}$, E. Focardi ${ }^{7}$, M. French ${ }^{14}$, K. Freudenreich ${ }^{16}$, A. Fürtjes ${ }^{6}$, A. Giassi ${ }^{13}$, A. Giraldo ${ }^{11}$, B. Glessing ${ }^{6}$, W.H. Gu${ }^{1}$, G. Hall ${ }^{9}$, R. Hammerstrom ${ }^{6}$, T. Hebbeker ${ }^{3}$, J. Hrubec ${ }^{15}$, M. Huhtinen ${ }^{6}$, A. Kaminsky ${ }^{11}$, V. Karimaki ${ }^{8}$, St. Koenig ${ }^{1}$, M. Krammer ${ }^{15}$, P. Lariccia ${ }^{12}$, M. Lenzi ${ }^{7}$, M. Loreti ${ }^{11}$, K. Luebelsmeyer ${ }^{1}$, W. Lustermann ${ }^{16}$, P. Mättig ${ }^{6}$, G. Maggi ${ }^{2}$, M. Mannelli ${ }^{6}$, G. Mantovani ${ }^{12}$, A. Marchioro ${ }^{6}$, C. Mariotti ${ }^{6}$, G. Martignon ${ }^{11}$, B. Mc Evoy ${ }^{9}$, M. Meschini ${ }^{7}$, A. Messineo ${ }^{13}, \mathrm{~S} \mathrm{My}^{2}$, A. Paccagnella ${ }^{11}$, F. Palla ${ }^{13}$, D. Pandoulas ${ }^{1}$, G. Parrini ${ }^{7}$, D. Passeri ${ }^{12}$, M. Pieri ${ }^{7}$, S. Piperov ${ }^{3}$, R. Potenza ${ }^{5}$, F. Raffaelli ${ }^{13}$, G. Raso ${ }^{2}$, M. Raymond ${ }^{9}$, F. Rizzo ${ }^{13}$, B. Schmitt ${ }^{6}$, G. Selvaggi ${ }^{2}$, L. Servoli ${ }^{12}$, G. Sguazzoni ${ }^{13}$, R. Siedling ${ }^{1}$, L. Silvestris ${ }^{2}$, K. Skog ${ }^{8}$, A. Starodumov ${ }^{13}$, I. Stavitski ${ }^{11}$, G. Stefanini ${ }^{6}$, P. Tempesta ${ }^{2}$, G. Tonelli ${ }^{13}$, A. Tricomi ${ }^{5}$, T. Tuuva ${ }^{10}$, C. Vannini ${ }^{13}$, P.G. Verdini ${ }^{13}$, G. Viertel ${ }^{16}$, Z. Xie ${ }^{13}$, Y. Wang ${ }^{12}$, S. Watts ${ }^{4}$, B. Wittmer ${ }^{1}$.

${ }^{1}$ I. Physikalisches Institut, RWTH, Aachen, Germany

${ }^{2}$ INFN sez. di Bari and Università di Bari, Bari, Italy

${ }^{3}$ Humboldt University, Berlin, Germany

${ }^{4}$ Brunel University, Middlesex, United Kingdom

${ }^{5}$ INFN sez. di Catania and Università di Catania, Catania, Italy

${ }^{6}$ CERN, European Laboratory for Particle Physics, Geneva, Switzerland

${ }^{7}$ INFN sez. di Firenze and Università di Firenze, Firenze, Italy

${ }^{8}$ Helsinki Institute of Physics, Helsinki, Finland

${ }^{9}$ Imperial College, United Kingdom

${ }^{10}$ University of Oulu, Kemi, Finland

${ }^{11}$ INFN sez. di Padova and Università di Padova, Padova, Italy

${ }^{12}$ INFN sez. di Perugia and Università di Perugia, Perugia, Italy

${ }^{13}$ INFN sez. di Pisa and Università di Pisa, Pisa, Italy

${ }^{14}$ Rutherford Appleton Laboratory, Didcot, United Kingdom

${ }^{15}$ Institut fur Hochenergiephysik der OeAW, Wien, Austria

${ }^{16}$ Laboratory for High Energy Physics, ETH, Zurich, Switzerland

*corresponding author, e-mail: concezio.bozzi@cern.ch

\section{Abstract}

We report selected results of laboratory measurements and beam tests of heavily irradiated microstrip silicon detectors. The detectors were single-sided devices, produced by different manufacturers and irradiated with different sources, for several total ionizing doses and fluences up to 4 $\times 10^{14} 1-\mathrm{MeV}$-equivalent neutrons per $\mathrm{cm}^{2}$. Strip resistance and capacitance, detector leakage currents and breakdown performance were measured before and after irradiations. Signal-to-noise ratio and detector efficiency were studied in beam tests, for different values of the detector temperature and of the read-out pitch, as a function of the detector bias voltage. The goal of these test is to optimise the design of the final prototypes for the Silicon Strip Tracker of the CMS experiment at the CERN LHC collider.

\section{INTRODUCTION}

The LHC at CERN, to be completed by 2005, will collide protons at $14 \mathrm{TeV}$ center-of-mass energy. The CMS detector at the LHC has been designed to detect cleanly the diverse signatures of new physics and, more generally, to perform precise measurements of physics processes at the TeV energy scale. Robust tracking and detailed vertex reconstruction within a strong magnetic field are powerful tools to reach these objectives. The CMS Tracker [1] will consist of three subsystems, the Pixel Detector System, the Silicon Strip Tracker, and the Micro-Strip Gas Chamber Tracker, each best matched to the task of satisfying the stringent resolution and granularity requirements in the regions of high, medium, and lower density of particles produced by the LHC collisions. The subsystems are arranged in concentric cylindrical volumes, each corresponding to the three occupancy regimes: $\approx 20 \mathrm{~cm}$, between $\approx 20$ and $\approx 60 \mathrm{~cm}$, and from $\approx 70 \mathrm{~cm}$ to $120 \mathrm{~cm}$ from 

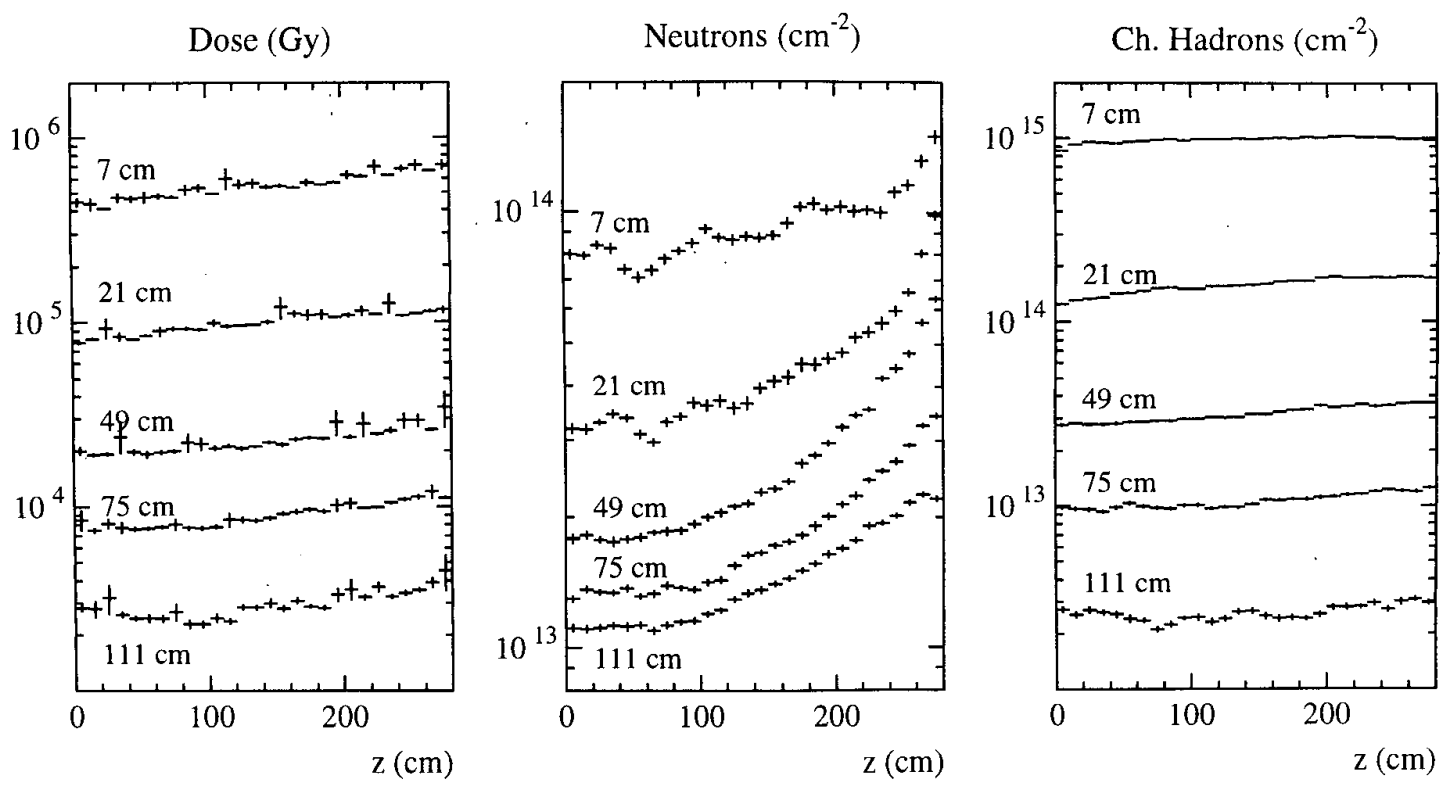

Figure 1: Radiation levels in the CMS Tracker region, at selected distances $(7,21,49,75,111 \mathrm{~cm})$ from the beam line. $z$ is the coordinate along the beam. The Silicon Strip Tracker extends between 22 and $60 \mathrm{~cm}$. All values correspond to an LHC integrated luminosity of $5 \times 10^{5} \mathrm{pb}^{-1}$.

the beam axis, respectively. The Silicon Strip Tracker (SST) [2] consists of approximately $70 \mathrm{~m}^{2}$ of silicon micro-strip detectors, arranged in a barrel and two end-cap regions, instrumenting the radial region between 22 and $60 \mathrm{~cm}$.

The most critical issue of the CMS Tracker is the long-term survival under heavy irradiation. Figure 1 shows the expected total ionizing dose, neutron and charged hadron fluences in the CMS Tracker region, at 5 different distances from the beam line, for an LHC integrated luminosity of $5 \times 10^{5} \mathrm{pb}^{-1}$. Secondary particles from the $p p$ interactions, the product of their interactions in the Tracker structure and some decay products give the dominating contribution to the fluences at the inner layers of the Tracker. This component is almost independent of the coordinate along the beam axis $(z)$ and behaves roughly as $1 / \dot{r}^{2}$, where $r$ is the distance from the beam line. Almost all of the charged hadron fluence originates from the vertex. On the other hand, most of the neutrons are due to albedo from the surrounding electromagnetic calorimeter, the most intense contribution coming from the calorimeter end-caps. In 10 years of LHC running the most irradiated detector of the Silicon Strip Tracker will receive a fluence of $1.6 \times 10^{14} 1-\mathrm{MeV}$-equivalent neutrons per $\mathrm{cm}^{2}$. A more detailed analysis, as well as a thorough discussion of the methods used for estimating the radiation levels, can be found in [3].

The technology for the radiation resistance of silicon micro-strip detectors has been thoroughly investigated by several groups [4]. The design of the micro-strip devices for the SST will be based on single-sided $p^{+}$implants in an initially $n$-type bulk silicon which offers a great advantage in terms of cost and industrial production capability. This choice, however, presents a significant challenge due to heavy radiation damage.

The radiation damage can be divided into two classes: surface damage and bulk damage. The former occurs when the holes produced by ionizing radiation in the surface oxide layer either becom trapped in the oxide or interact with atoms at the silicon-oxide interface to form interface states. Fixed positive charge in the oxide modifies the oxide field, while interface states give rise to new energy levels in the forbidden gap which can modify device behaviour [5]. These changes can lead to a decrease in the inter-strip isolation, causing unwanted signal charge sharing and increase in the inter-strip capacitance, which is the major contribution to the electronic noise of the system. The bulk damage is due to hadrons, and leads to the creation of defects in the silicon lattice. This affects the leakage current, which increases proportionally to the received fluence and is strongly dependent on the temperature [6]. Another effect of the bulk damage concerns the decrease in charge collection efficiency caused by the charge trapping at radiation-induced levels in the silicon bulk [7]. It is estimated [6] that the resulting ballistic deficit will be tolerable.

The bulk damage also influences the effective doping 
Table 1

Detector specifications. Several samples of each detector type were each irradiated once at the specified fluence.

\begin{tabular}{|c|c|c|c|c|c|c|c|c|}
\hline & \multicolumn{4}{|c|}{ Strip parameters } & \multicolumn{3}{|c|}{ Detector parameters before irradiation } & Irradiation \\
\hline Manufacturer & $\begin{array}{c}\text { Lenght } \\
(\mathrm{cm})\end{array}$ & $\begin{array}{l}\text { Pitch } \\
(\mu \mathrm{m})\end{array}$ & $\begin{array}{l}\text { Width } \\
(\mu \mathrm{m})\end{array}$ & $\begin{array}{l}\text { Number } \\
\text { of Strips }\end{array}$ & $\begin{array}{l}\text { Depletion } \\
\text { Voltage (V) }\end{array}$ & $\begin{array}{l}\text { Breakdown } \\
\text { Voltage(V) }\end{array}$ & $\begin{array}{c}\text { Leakage } \\
\text { Current }(\mathrm{nA})\end{array}$ & $\begin{array}{c}\text { Fluence }\left(10^{14} \mathrm{~cm}^{-2}\right) \\
\text { and conditions }\end{array}$ \\
\hline $\begin{array}{c}\text { CSEM } \\
\text { [11] }\end{array}$ & 6.25 & $\begin{array}{l}50 \\
60 \\
75\end{array}$ & $\begin{array}{c}10 \\
10 \\
10,30,50\end{array}$ & 1024 & 50 & $\begin{array}{c}\text { Mean Value: } \\
580 \pm 200\end{array}$ & 50 & $\begin{array}{l}\text { Neutrons [13]: } \\
0.1,0.9,1.8,2.7,3.6 \\
\mathrm{~T}=20^{\circ} \mathrm{C}, \mathrm{Bias} \text { OFF }\end{array}$ \\
\hline $\begin{array}{c}\text { HAMAMATSU } \\
\text { [12] }\end{array}$ & 6.25 & $\begin{array}{c}60 \\
80 \\
120 \\
240\end{array}$ & $\begin{array}{l}15,20,25 \\
15,25,40 \\
20,35,50 \\
20,50,70\end{array}$ & 32 & 60 & $>600$ & $<200$ & $\begin{array}{l}\text { Protons: } \\
.18, .35, .96 \\
\text { Neutrons }[14]: \\
0.05,0.17,0.31,1.7 \\
\mathrm{~T}<-8^{\circ} \mathrm{C}, 150 \mathrm{~V} \text { bias }\end{array}$ \\
\hline
\end{tabular}

concentration of the substrate, which is determined by the concentration of space charge in the depletion region. Since irradiation results in an accumulation of negative space charge in the depletion region due to the introduction of acceptor defects which have energy levels deep within the forbidden gap, $n$-type detectors become progressively less $n$-type with increasing hadron fluence until they invert to effectively $p$-type and then continue to become more $p$-type beyond this point, apparently whitout limit $[8,9]$. The inversion fluence depends strongly on the initial resistivity of the substrate material. Detectors still work beyond the inversion fluence as the junction moves from the $p^{+}$strips to the $n^{+}$backplane contact. At high fluences, however, the effective doping concentration can be such as the depletion voltage exceeds the breakdown voltage of the device and efficient operation is no longer possible. In any case, the detectors must be substantially over-depleted in order to maintain a satisfactory performance. Since the expected depletion voltage after type-inversion increases rapidly as a function of the fluence, the detectors must be designed to allow for high voltage $(\approx 500 \mathrm{~V})$ operation. In addition, to limit the effect of the radiation damage, silicon detectors should be operated at low temperature. The entire volume of the SST will be kept at $-10^{\circ} \mathrm{C}$ during the LHC running $[2,9,10]$.

Several test structures and detector prototypes were used to validate the design choices and processing technologies for the detectors foreseen for the SST. Here we will present a selection of the results for single-sided, $p^{+}$on $n$-bulk, 300 $\mu \mathrm{m}$ thick, high bulk resistivity ( $>6 \mathrm{k} \Omega \cdot \mathrm{cm}$ ), AC-coupled, polysilicon-biased devices, with two guard rings. Other detector parameters are summarized in table I. The detectors were manufactured by CSEM [11] and Hamamatsu [12]. Irradiations were performed with fast neutrons from nuclear reactors $[13,14]$ and with $24-\mathrm{GeV}$ protons from the CERN PS. The irradiation conditions such as detectors bias voltage and temperature are also summarized in table I. The energy spectrum of the neutrons is in the $\mathrm{MeV}$ range, and is very similar to the one expected for LHC. Fluences were properly converted into $1-\mathrm{MeV}$-equivalent-neutrons units. Detectors were kept below $0{ }^{\circ} \mathrm{C}$ after irradiation. It should be noted that irradiations at nuclear reactors imply the presence of gamma rays in the $\mathrm{MeV}$ energy range from nuclear reactions, which are responsible for the surface damage of the detectors. The corresponding total ionization dose was not measured, but we estimate from the high neutron fluxes that the associated dose from gamma rays was high enough to produce a saturation in the density of trapped oxide charges at the $\mathrm{Si}_{-} \mathrm{SiO}_{2}$ interface.

\section{LABORATORY MEASUREMENTS ON PROTOTYPES}

\section{A. Strip Resistance and Capacitance}

The resistance between $p^{+}$strips is very high $(>100 \mathrm{M} \Omega$ ) before irradiation. After type inversion lower values (10-100 $\mathrm{M} \Omega$ ) for the inter-strip resistance have been observed for all the detectors studied. However, the contribution to the detector noise [15] is still negligible.

The strip series resistance depends on the geometry and the resistivity of the metal line. Standard aluminium-silicon resistivity has been measured around $27 \mathrm{~m} \Omega \cdot \mu \mathrm{m}$, which results in a series resistance of the order of $90 \Omega$ for metal electrodes $12 \mu \mathrm{m}$ wide, $1.5 \mu \mathrm{m}$ thick and $62.5 \mathrm{~mm}$ long.

The capacitive coupling of each strip to the adjacent ones is determined by the shape of the electric potential between the $p^{+}$implants and is strongly influenced by the fixed charge at the $\mathrm{Si}-\mathrm{SiO}_{2}$ interface. The role of various designs and technologies has been investigated before and after irradiation. The experimental setup used to measure the capacitive couplings between strips is described in [15]. Figure 2 shows the value of the total capacitive coupling per unit length,

$$
C_{t o t}=C_{b}+2\left(C_{1 n}+C_{2 n}\right)
$$

as a function of the strip width over pitch ratio. $C_{b}$ is the capacitance to the back, while $C_{1 n}$ and $C_{2 n}$ describe the couplings to the first and the second neighbouring strips, respectively. The measurements were performed at $100 \mathrm{~V}$ bias voltage and $1 \mathrm{MHz}$ frequency on detectors with high resistivity (> $6 \mathrm{k} \Omega \cdot \mathrm{cm}$ ) substrates. This bias voltage was enough for the detector to be substantially over-depleted and for the 


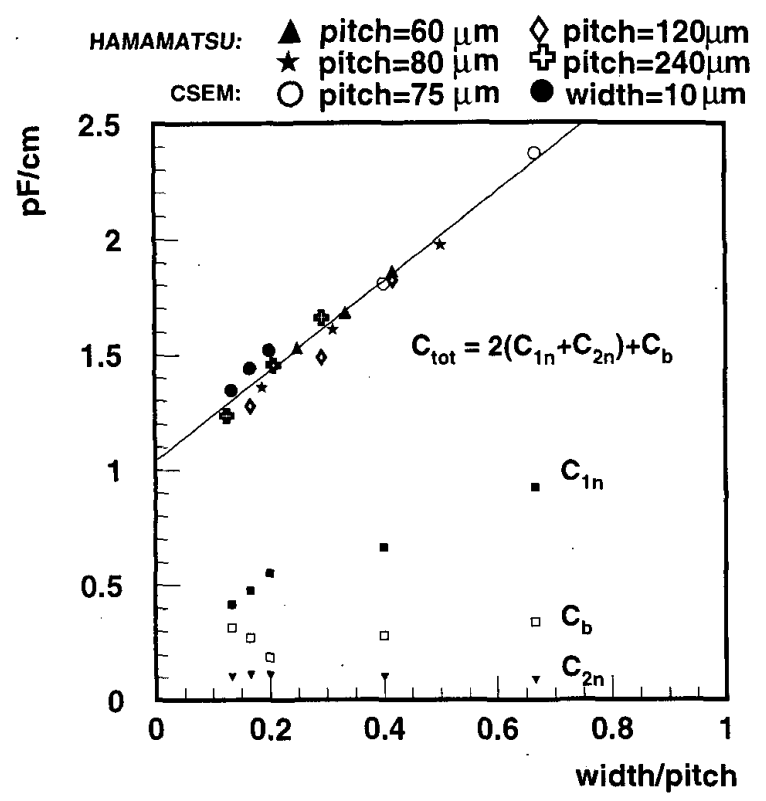

Figure 2: Total capacitance per unit length as a function of strip width/strip pitch ratio $(\mathrm{w} / \mathrm{p})$ for non-irradiated detectors from Hamamatsu and CSEM.

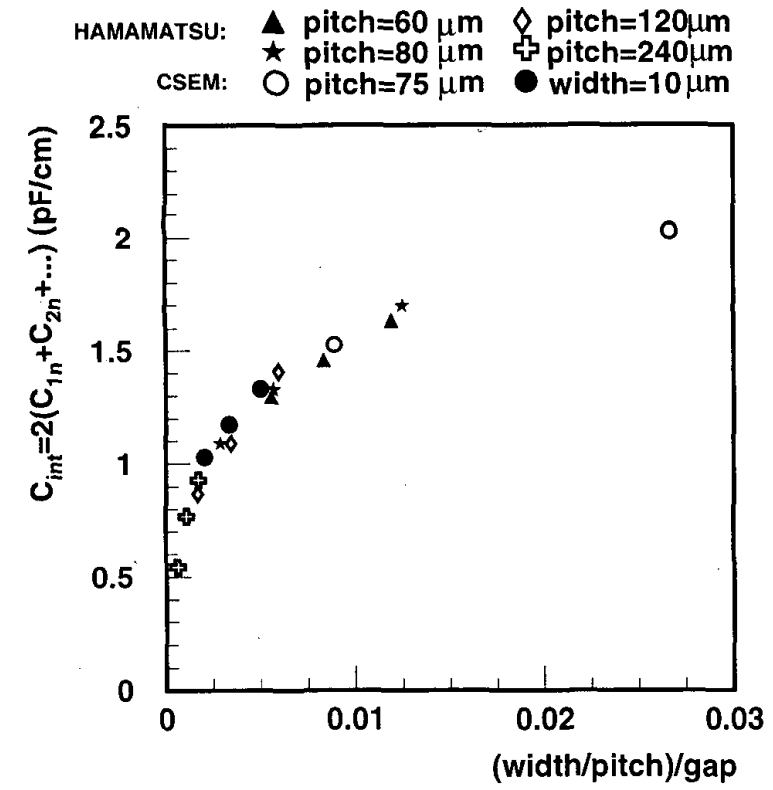

Figure 3: Total inter-strip capacitance $\mathrm{C}_{i n t}=2 \sum_{i} \mathrm{C}_{i n}$ per unit length, as a function of the $w / p$ over gap ratio. The gap is the difference between strip pitch and width. Same detectors as of Fig. 2

capacitances to reach an asymptotic value. The total capacitive load is dominated by $C_{1 n}$ and, to a good approximation, is a linear function of the strip width over pitch ratio. A good parametrisation of the total inter-strip capacitance per unit length, $\mathrm{C}_{i n t}=2 \sum_{i} \mathrm{C}_{i n}$, can be obtained for different designs and technologies by plotting it (Figure 3 ) as a function of the width/pitch ratio divided by the gap between $p^{+}$implants.
The concentration of positive fixed charge in the oxide increases up to the saturation value with irradiation. The higher concentration of negative charge at the interface results in a stronger inter-strip coupling. A reduction of inter-strip capacitance can be obtained in all devices by increasing the bias voltage, since a higher field on the $p^{+}$side confines the electrons at the interface to the middle of the gap between two implants. Figure 4 shows the inter-strip capacitance versus the bias voltage for CSEM detectors after neutron irradiations [15]. As already mentioned before, the neutrons, responsible
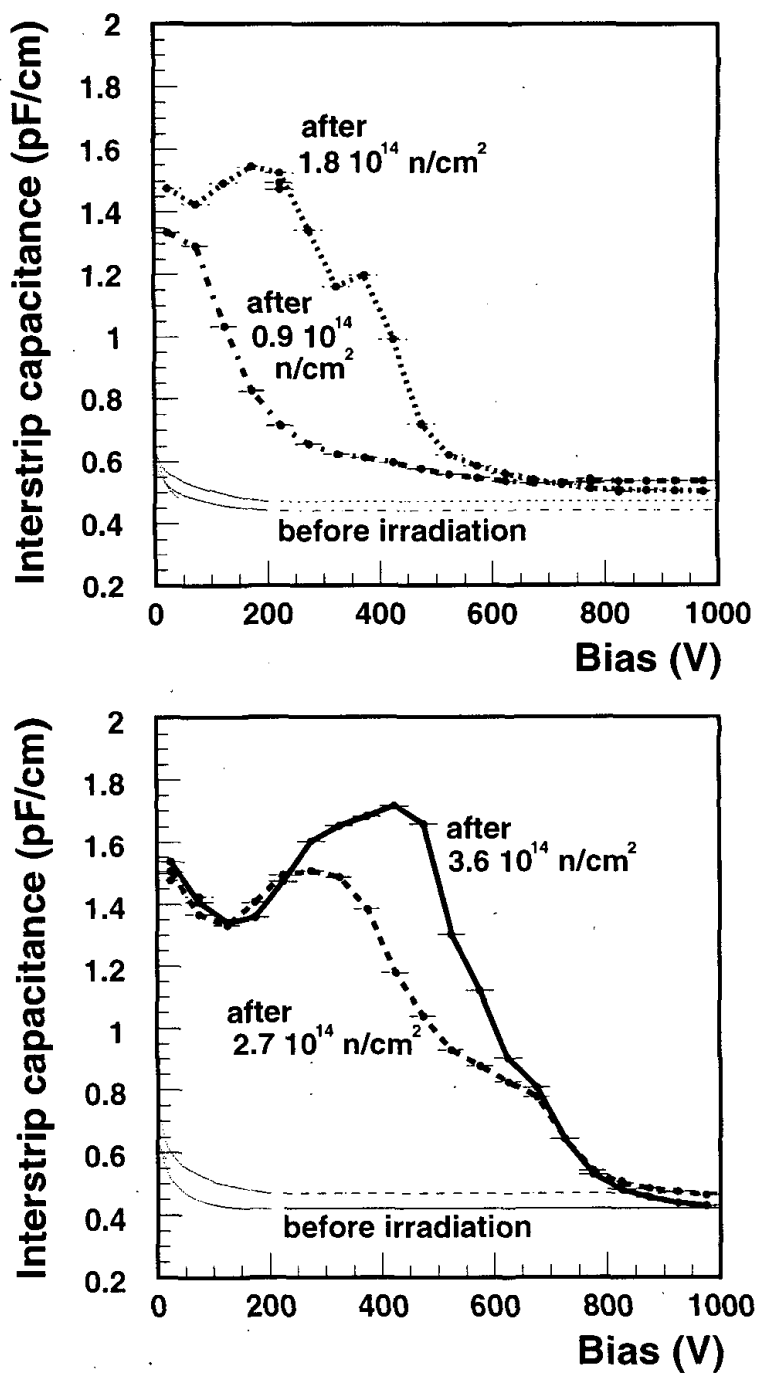

Figure 4: Capacitance between first neighbouring strips before and after different neutron irradiations as a function of the bias voltage. Detectors manufactured by CSEM.

for the bulk damage, were accompanied by gamma rays in the $\mathrm{MeV}$ energy range, which were responsible for the surface damage, thus for the change in the inter-strip capacitance. The detectors were at $20^{\circ} \mathrm{C}$ temperature and they were not biased 
during the irradiation. The inter-strip capacitances measured after irradiation show a very strong bias dependence. At full depletion the coupling is still high but it sharply decreases by increasing the bias voltage. Asymptotic values at most $25 \%$ higher with respect to the pre-irradiation values can be reached for a bias voltage 1.5 higher than the depletion voltage.

The possibility of minimizing the effects of both the bulk and the surface damage, by using detectors with low resistivity $(1 \mathrm{k} \Omega \cdot \mathrm{cm})$ substrates and $\langle 100\rangle$ lattice orientation, is currently under study.

\section{B. Breakdown Performance}

The breakdown characteristics of several series of devices from different manufacturers have been measured before and after irradiation. Breakdown voltage distribution for devices (CSEM) before irradiation is shown in figure 5. Most of the detectors fulfil the requirement of breakdown voltage above $500 \mathrm{~V}$. Similar performances are obtained after proton and neutron irradiation. As an example, figure 6 shows the leakage current as a funcion of bias voltage after neutron irradiation for Hamamatsu detectors, at $-10{ }^{\circ} \mathrm{C}$ temperature. Measured values of the leakage current at maximum bias voltage after irradiation are compatible with the maximum tolerable leakage current of $25 \mu \mathrm{A} / \mathrm{cm}^{2}$ at a temperature of $-10^{\circ} \mathrm{C}$ [2]. Detector leakage current variations are linear with the fluence. The damage constant $\alpha$ normalised at $20^{\circ} \mathrm{C}$ is $(4 \pm 1) \cdot 10^{-17} \mathrm{~A} / \mathrm{cm}$, to be compared with an expected value of $2.9 \pm 0.2 \cdot 10^{-17} \mathrm{~A} / \mathrm{cm}$ [16].

\section{Possible Design Improvements}

Laboratory measurements indicate some problems of the simple edge design ( $n$-well on the cutting area and two guard-rings) we have adopted for all the detectors tested in this work. When a high reverse bias is applied to both the detector active area and the external guard-ring, intense electric fields may lead to avalanche breakdown on the outer side of the external guard-ring causing physical breakdown of the device.

Another problem is related with surface generated leakage current and carrier generation current from the edge, where generation-recombination centers are induced by, cutting. This current is a source of extra noise. With the simple edge design the external guard-ring collects the surface current, while the n-well creates a barrier for hole injection.

Improvements can be achieved by shaping the electric field using a series of floating $\mathrm{p}^{+}$rings (a multi-guard structure) around the guard-ring. A particular scheme (figure 7) has been tested making use of multi-guard structures, optimised by means of device characterisation, irradiation tests and simulations $[17,18]$. The overall results may be used to give prescriptions on the geometrical arrangement and doping of the multi-guard structure in order to optimise the breakdown behaviour [19].

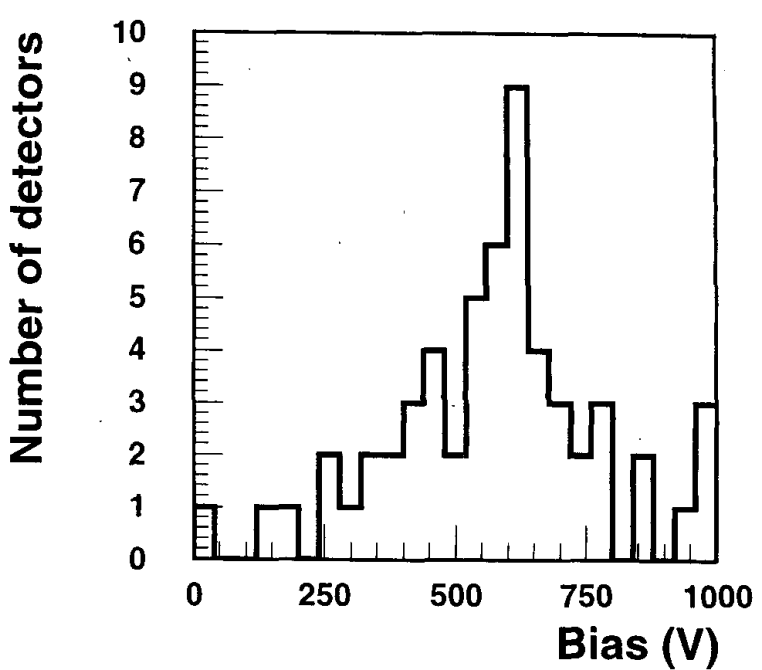

Figure 5: Breakdown voltage values for full-size, non-irradiated detectors produced by CSEM. The average value is at $580 \mathrm{~V}$ with a r.m.s. spread of $200 \mathrm{~V}$

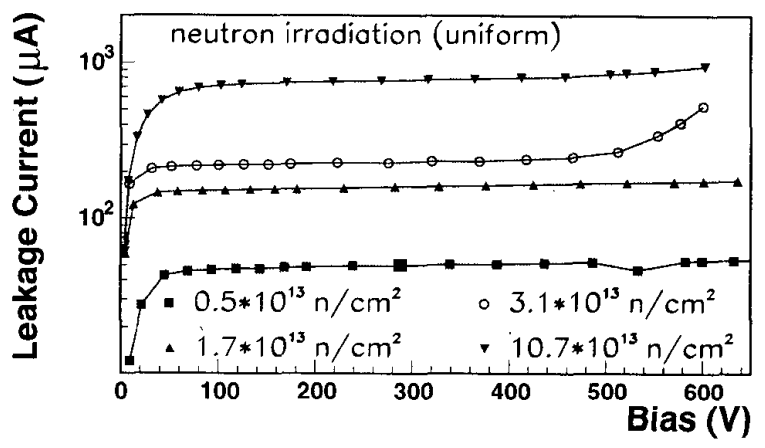

Figure 6: $I-V$-curves for $3 \times 6 \mathrm{~cm}^{2}$ devices after uniform neutron irradiation, for Hamamatsu detectors, at $-10^{\circ} \mathrm{C}$ temperature.

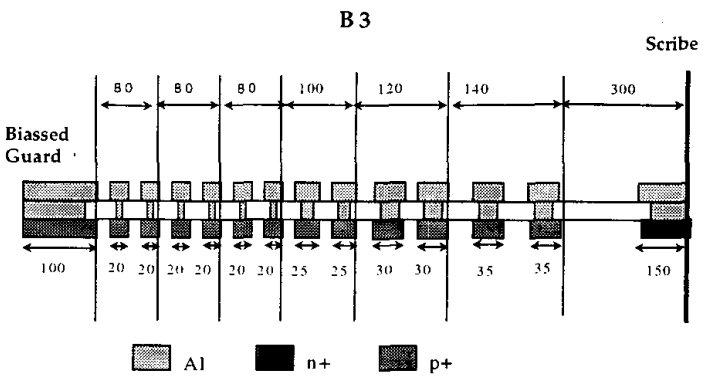

Figure 7: Cross-section of a possible multi-guard structure layout. The biased guard ring is on the left, while the scribe line is on the right. Distances are expressed in microns. 


\section{TEST BEAM RESULTS}

A series of beam tests has been carried out on heavily irradiated detectors. Here we will present results obtained with the $100 \mathrm{GeV}$ muon beam of the X5 CERN experimental area. The silicon sensors were mounted in detector modules, formed by one or two silicon detectors, and a read-out hybrid, which houses the front-end electronics [20]. Each micro-strip is read out by a fast charge sensitive preamplifier, wire-bonded to the strip, followed by a shaper, with peak mode readout and $50 \mathrm{~ns}$ shaping time. The strip length varies from 6.25 to $12.5 \mathrm{~cm}$, depending on whether a module consists of one or two silicon sensors. In the latter case, the micro-strips of the two sensors were wire-bonded to form a single electrical unity. The modules under test were inserted in a "cold box", in which cooled nitrogen was circulated and where the temperature could be varied from $-20^{\circ} \mathrm{C}$ to room temperature; the temperature, monitored inside the box, was stable within $\pm 0.5^{\circ} \mathrm{C}$. The box was inserted in the middle of a silicon telescope made of four planes of double-sided microstrip detectors [21]. This telescope allows a tri-dimensional tracks reconstruction with a resolution in both coordinates $\leq 4.0$ $\mu \mathrm{m}$. The set of algorithms used for cluster finding and for determining the cluster charge and noise are described in [22]. Pedestal, single-channel noise, common-mode noise, signal common-mode-subtracted and cluster selection were calculated on an event-by-event basis using pre-determined thresholds.

\section{A. Signal-to-Noise Ratio}

Signal-to-Noise $(\mathrm{S} / \mathrm{N})$ is defined as the ratio of the most probable value of the Landau fit to the cluster signal distribution with the average single strip noise. The most important noise contributions in our detectors come from: the thermal noise, due to the strip and bias resistances; the amplifier noise, seen as a noise source applied to the input of the amplifier, which depends linearly from the capacitive load seen at the amplifier input towards the detector. An estimation of the relative contribution to the noise of the system can be found in [23]. The influence of the strip pitch and width on the $\mathrm{S} / \mathrm{N}$ ratio has been investigated. Non-irradiated detectors from Hamamatsu with different readout pitch values, ranging from 60 to $240 \mu \mathrm{m}$ and comparable width/pitch ratios (0.21-0.33), show similar behaviour of $\mathrm{S} / \mathrm{N}$ as a function of bias voltage (figure 8), This because the noise is dominated by amplifier noise, that depends on the total capacitance, which is (figure 2) nearly constant for the set of detectors of figure 8 . Similar performance was observed also for CSEM detectors.

Since in a typical LHC collision a significant fraction of particles will cross the detectors at non-orthogonal incident angles, the effect of inclined tracks has been studied in detail. The amount of charge released in the silicon and the number of fired strips is expected to increase while the average single strip noise should remain unaffected. The observed $\mathrm{S} / \mathrm{N}$ ratio is compatible with the predicted $1 / \cos \theta$ dependence of the cluster charge and a constant strip noise [2].

The surface radiation damage produces an increase of the noise, as a consequence of the increased inter-strip

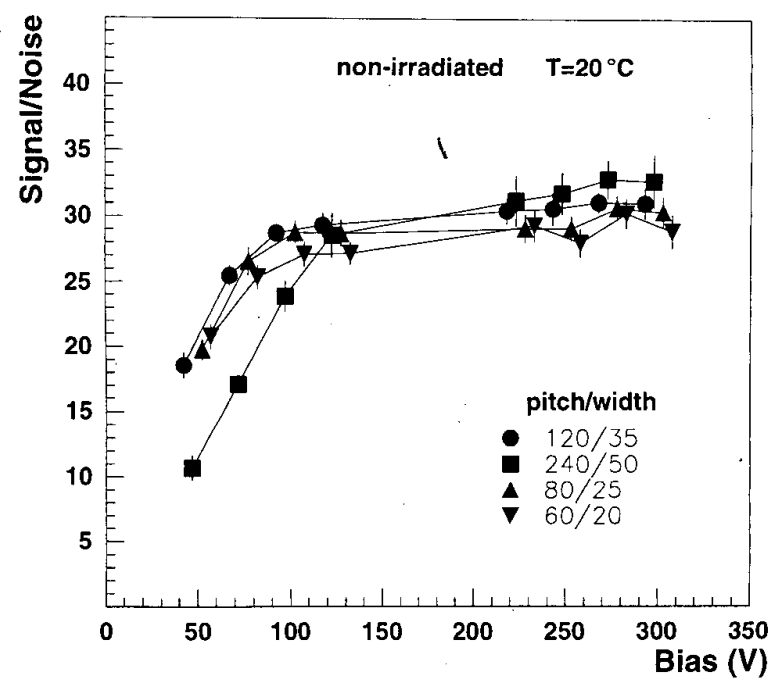

Figure 8: Signal-to-noise ratio vs bias voltage. Non-irradiated Hamamatsu detectors with strip pitches varying from $60 \mu \mathrm{m}$ to $240 \mu \mathrm{m}$. Strip length is $6.5 \mathrm{~cm}$.

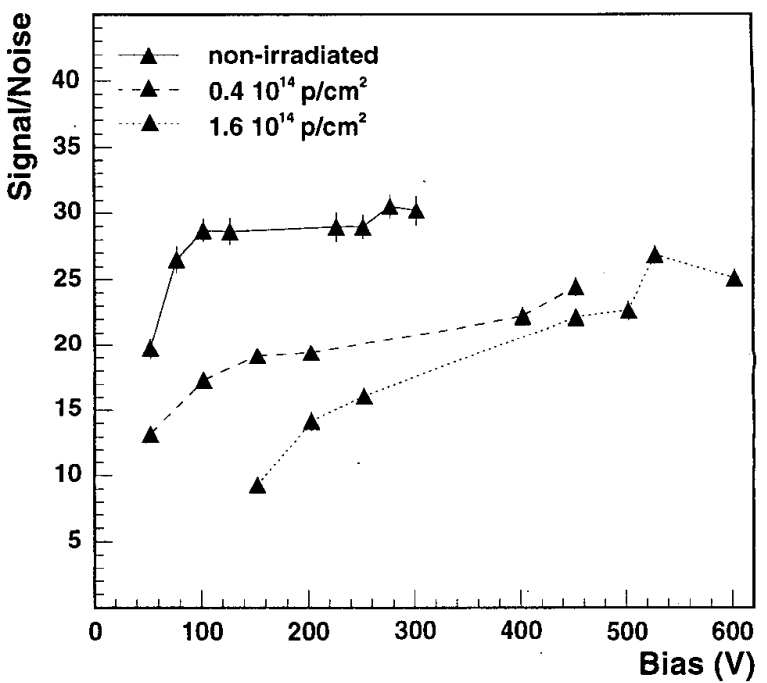

Figure 9: Signal-to-noise ratio vs bias voltage. Hamamatsu detectors irradiated with neutrons. The strip pitch is $80 \mu \mathrm{m}$, the strip length is $6.5 \mathrm{~cm}$.

capacitance (Figure 4), while the bulk damage causes a decrease of the charge collection efficiency [15]. These two effects can be partly reduced by increasing the bias voltage above the full depletion value. The $\mathrm{S} / \mathrm{N}$ ratio for irradiated devices approaches a plateau value at high bias voltages. Figure 9 shows the $\mathrm{S} / \mathrm{N}$ ratio as a function of bias voltage for Hamamatsu detectors irradiated with $24 \mathrm{GeV}$ protons and fluences up to $1.6 \times 10^{14} \mathrm{p} / \mathrm{cm}^{2}$. Figure 10 shows the $\mathrm{S} / \mathrm{N}$ as a function of the bias voltage in over-depletion units for two non-irradiated detectors (made by CSEM and SGS [25]) and two CSEM detectors irradiated with neutrons at fluences of 1 and $2 \times 10^{14}$ neutrons per $\mathrm{cm}^{2}$. The detectors were kept at $-10^{\circ} \mathrm{C}$ temperature. The depletion voltage is around $60 \mathrm{~V}$ 


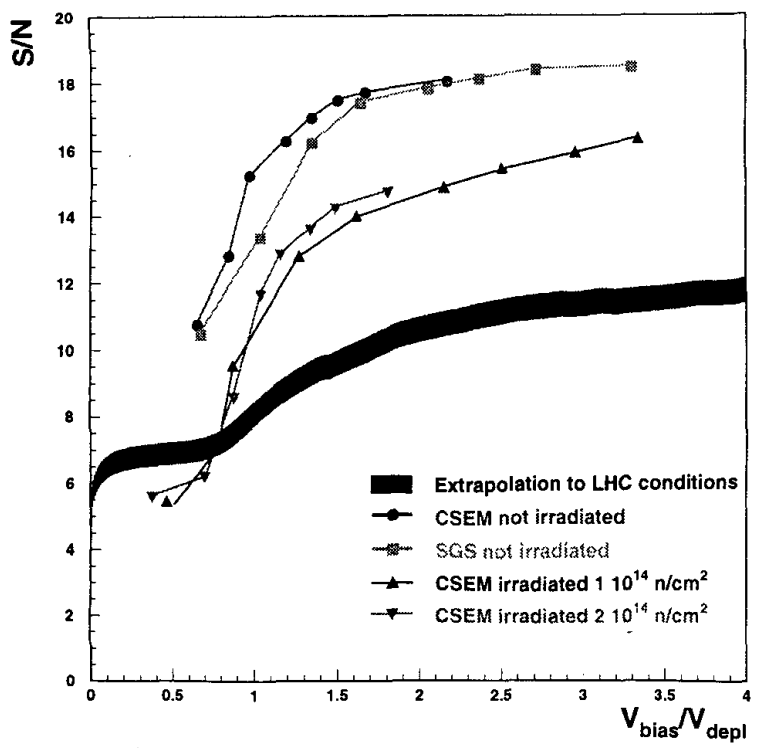

Figure 10: Signal-to-noise ratio as a function of bias voltage in over-depletion units, for two non-irradiated detectors (CSEM, SGS), and two detectors (CSEM) irradiated at $1 \times 10^{14} \mathrm{n} / \mathrm{cm}^{2}$ and $2 \times 10^{14} \mathrm{n} / \mathrm{cm}^{2}$, respectively. The readout pitch is $50 \mu \mathrm{m}$, the strip length is $12.5 \mathrm{~cm}$. The operating temperature is $-10^{\circ} \mathrm{C}$. The blue band is an analytical extrapolation (see text) to the experimental conditions foreseen at the LHC.

for the non-irradiated detectors, $120 \mathrm{~V}$ and $300 \mathrm{~V}$ for the two irradiated detectors. The over-depletion for the two irradiated detectors is calculated with respect to the depletion voltage after irradiation. There is still a $20 \%$ loss of signal-to-noise that is not recovered even going to the highest bias voltage. An analytical extrapolation to the expected LHC conditions, discussed in detail in [15] and confirmed by numerical simulations based on the SPICE program [24], is shown as a band in figure 10. The analytical model takes into account the performance of the final front-end chip [26]. The main differences with respect to a beam test setup will be the use of a deconvolution mechanism [27] to extract the signal and a slightly worse noise performance. The effect of proton irradiation has also been studied and leads to similar results.

Another important parameter which determines the behaviour of the detectors is the temperature at which the silicon is operated. This influences both the amount of charge collected and the noise. An increase in the $S / N$ ratio up to $20 \%$ has been observed for irradiated detectors going from room temperatures to the operating temperature of $-10^{\circ} \mathrm{C}$. As an example, figure 11 shows the $\mathrm{S} / \mathrm{N}$ ratio as a function of temperature for various bias voltages, for a CSEM detector irradiated with neutrons at $1 \times 10^{13} \mathrm{n} / \mathrm{cm}^{2}$. A similar behavoiur is observed also for Hamamatsu detectors, and for higher fluences.

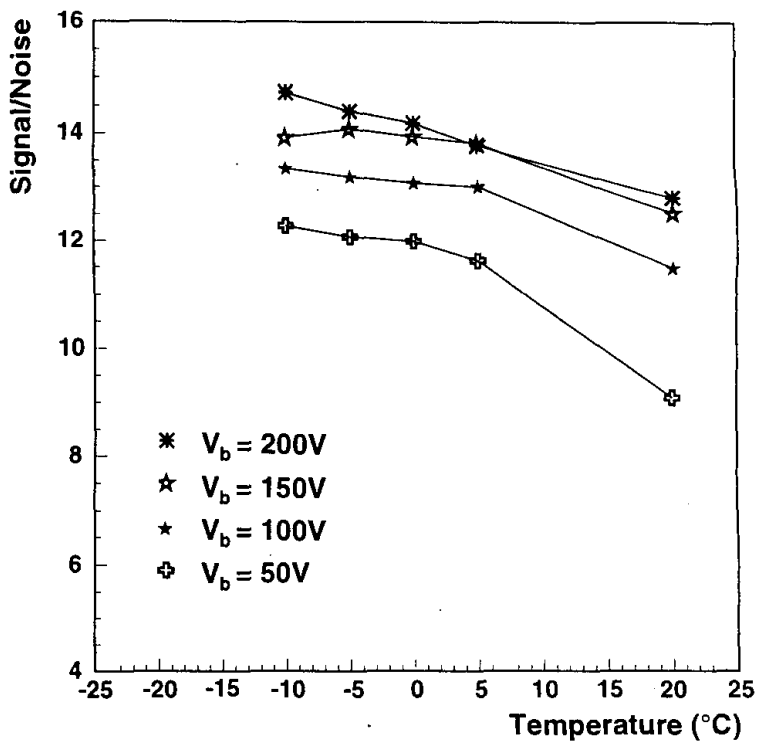

Figure 11: Signal-to-noise ratio as a function of temperature at various bias voltages for a detector from CSEM irradiated at $1 \times 10^{13} \mathrm{n} / \mathrm{cm}^{2}$. The readout pitch is $50 \mu \mathrm{m}$, the strip length is $11 \mathrm{~cm}$.

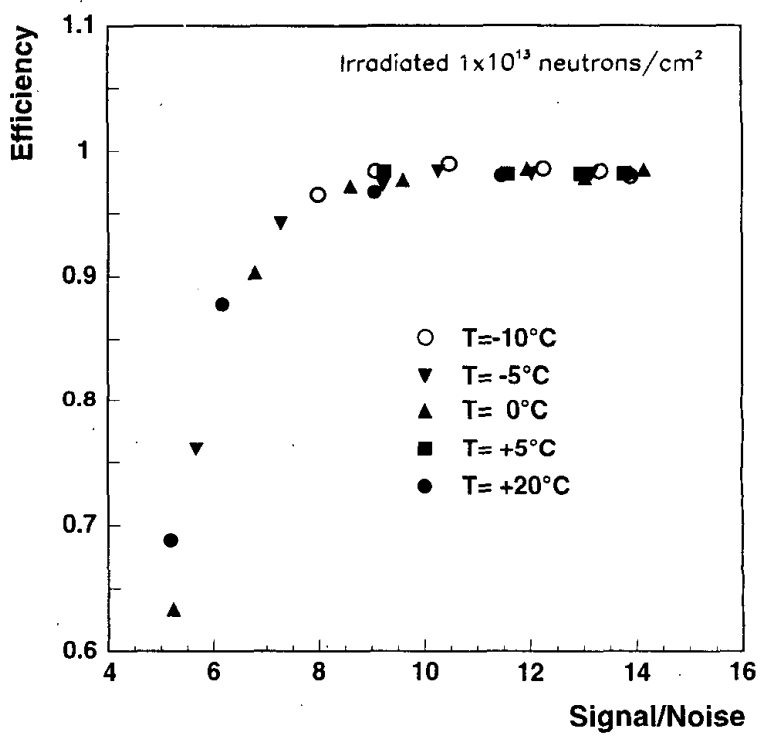

Figure 12: Efficiency vs. S/N for irradiated CSEM detectors of $50 \mu \mathrm{m}$ pitch, $6.22 \mathrm{~cm}$ length, at various temperatures.

\section{B. Detector Efficiency}

The global hit efficiency is defined as the ratio between the number of reconstructed and predicted hits. The latter are calculated from the number of tracks crossing the detector within its geometrical acceptance and away from dead or noisy strips. Figure 12 shows the dependence of this efficiency on signal-to-noise ratio at various temperatures for detectors irradiated with neutrons at $1 \times 10^{13} \mathrm{n} / \mathrm{cm}^{2}$, for particles impinging orthogonally to the detector surface.

It is worthwhile to note that in Figure 12 even when $\mathrm{S} / \mathrm{N}$ is 


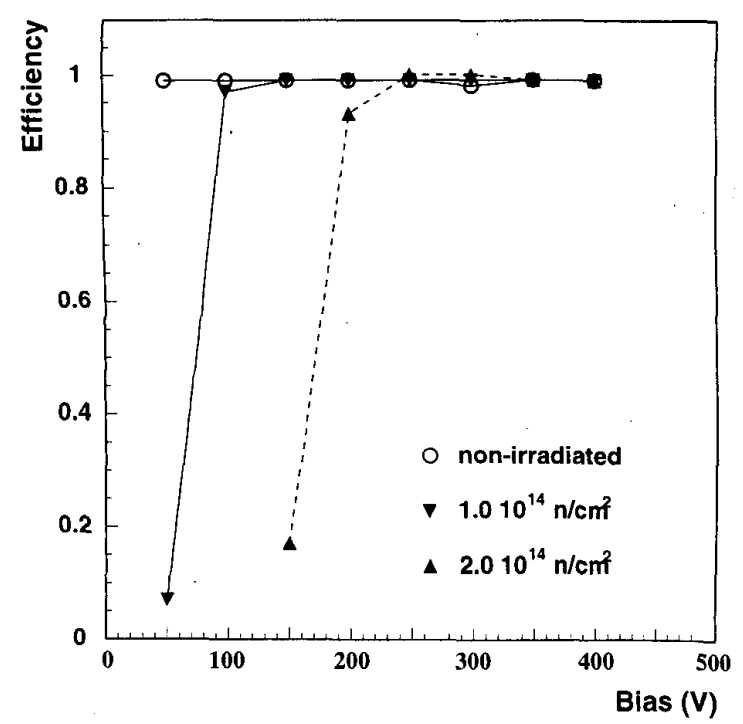

Figure 13: Efficiency as a function of bias voltage. CSEM detectors of $75 \mu \mathrm{m}$ pitch, $11 \mathrm{~cm}$ length, at $-10^{\circ} \mathrm{C}$ temperature, irradiated with neutrons.

7.5 we have an efficiency greater than $95 \%$. Figure 13 shows the dependence of the efficiency on bias voltage for a CSEM nonirradiated detector and for two CSEM detectors irradiated at $1 \times$ $10^{14} \mathrm{n} / \mathrm{cm}^{2}$ and $2 \times 10^{14} \mathrm{n} / \mathrm{cm}^{2}$, respectively. At full depletion, the efficiency is compatible with $100 \%$ for both irradiated and non-irradiated detectors.

The charge collection efficiency has been measured as a function of cluster position between two strips for different levels of radiation. The cluster charge distribution indicates good uniformity of charge collection even in heavily irradiated devices (Figure 14).

The measurement of ghosts, namely the fraction of hits not associated with tracks, has also been performed. A high ghost rate could spoil the pattern recognition task and the track finding efficiency. Values well below $1 \%$ were obtained, which is considered tolerable. The ghost rates do not depend on the operating conditions such as bias voltage and irradiation level [2].

Both the response function and the spatial resolution have been studied in a wide range of detectors. The experimental data show no significant discrepancy with respect to the expected performance [2].

\section{CONCLusion}

A wide $R$ \& $D$ program has been developed since a few years with the aim of assessing the performance of detectors suitable for the CMS Silicon Strip Tracker. The selected results presented in this paper confirm that the design options chosen so far can meet the requirements needed for CMS experiment. Laboratory measurements and beam test results, carried out before and after irradiation, confirm good performance of the current detectors design. Standard detector production shows acceptable performance on breakdown voltage values before

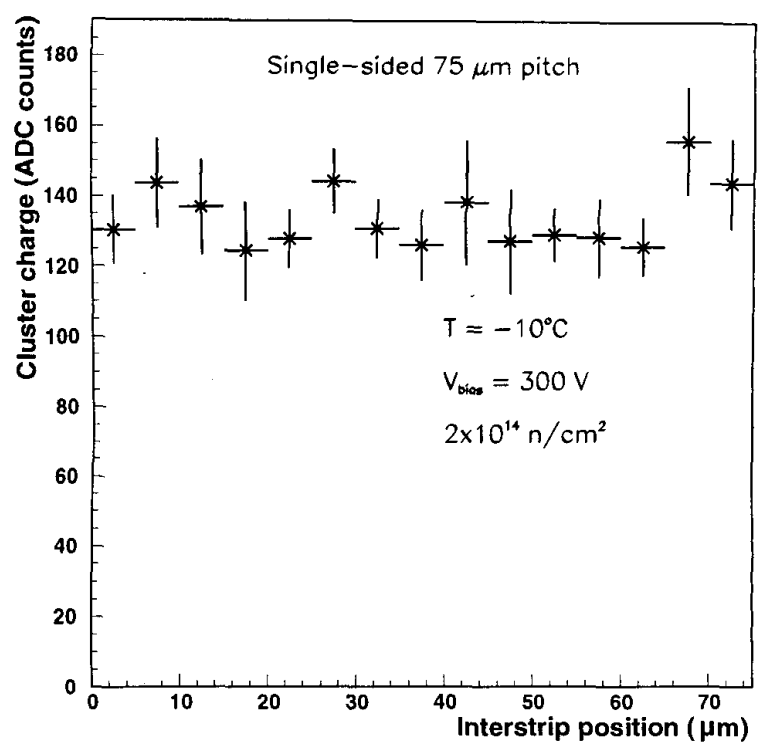

Figure 14: Average cluster charge as a function of the inter-strip coordinate in a CSEM device irradiated at $2 \times 10^{14} \mathrm{n} / \mathrm{cm}^{2}$. The strip pitch is $75 \mu \mathrm{m}$, the strip length is $11 \mathrm{~cm}$.

and after irradiation allowing a safe high voltage operation. Nevertheless a more efficient breakdown protection is foreseen with the use of multi-guard solution.

The behaviour of total strip capacitance has been well understood in terms of detectors geometry, and the effect of radiation damage has been investigated. After irradiation, performances similar to non irradiated detectors can be reached using over depletion bias voltage.

Signal to noise ratio has been studied in beam tests with different detectors geometry. The operation under real conditions shows good performance of detectors produced by different manufacturers. Besides measurements also analytical studies have been carried out. Extrapolation to the expected LHC condition shows acceptable $\mathrm{S} / \mathrm{N}$ value width a hit efficiency greater than $95 \%$.

\section{REFERENCES}

[1] The CMS Collaboration "The CMS Tracker Project Technical Design Report," CERN/LHCC 98-6, CMS TDR 5, 15 April 1998.

[2] The CMS Collaboration "The CMS Tracker Project Technical Design Report," CERN/LHCC 98-6, CMS TDR 5, 15 April 1998, Chapter 3, pp. 81-170, and references therein.

[3] The CMS Collaboration "The CMS Tracker Project Technical Design Report," CERN/LHCC 98-6, CMS TDR 5, 15 April 1998, Appendix A, pp. A.1-A.22, and references therein.

[4] RD2 Collaboration, "Radiation-induced bulk damage in silicon detectors", Nuclear Instruments and Methods in Phys. Res. A, vol. 383, 1996 p. 144.

RD20 Collaboration, RD20 Status Report 1995, 
CERN/LHCC/96-2 (1996)

RD48 Collaboration, RD48 Status Report, CERN/LHCC/97-39 (1997)

G. Tonelli et al. "Double-sided radiation resistant micro-strip detectors: technology and results," Nuclear Instruments and Methods in Phys. Res. A, vol. 377, 1996 p. 422.

[5] S.M. Sze "Semiconductor devices: physics and technology", Wiley, 1985, pp.379-407

[6] The CMS Collaboration "The CMS Tracker Project Technical Design Report," CERN/LHCC 98-6, CMS TDR 5, 15 April 1998, Chapter 3, pp. 94-97, and references therein.

[7] D. Passeri et al. "TCAD-Based Analysis of Radiation Hardness in Silicon Detectors", IEEE Transactions on Nuclear Science 45 (1998).

[8] K. Gill et al. "Radiation Damage by Neutrons and Photons to Silicon Detectors", Nuclear Instruments and Methods in Phys. Res. A, vol. 322, 1992 p. 177.

[9] F. Lemeilleur et al. "Study of characteristics of silicon detectors irradiated with $24 \mathrm{GeV} / c$ protons between $-20^{\circ} \mathrm{C}$ and $+20^{\circ} \mathrm{C}, "$ Nuclear Instruments and Methods in Phys. Res. A, vol. 360, 1995 pp. 438-444.

[10] E. Fretwurst et al. "Reverse annealing of the effective impurity concentration and long term operational scenario for silicon detectors in future collider experiments," Nuclear Instruments and Methods in Phys. Res. A, vol. 342, 1994 pp. 119-125.

[11] CSEM, Centre Suisse d'Electronique et Microelectronique, Neuchatel, Switzerland.

[12] Hamamatsu Photonics Co., Hamamatsu 435, Japan.

[13] Reattore Nucleare TAPIRO, ENEA, Centro Ricerche Casaccia, Roma, Italy.

S. Baccaro et al. "Gamma and neutron irradiation facilities at ENEA - Casaccia Center, Roma", CERN CMS NOTE TN/95.

[14] Ulysse heavy water reactor, CEA-Saclay, Gif-sur-Yvette, France.

[15] C. Bozzi et al. "Characterization and simulation of LHCtype silicon micro-strip detectors," Il Nuovo Cimento A, vol. 112,1999 p. 67.

[16] A. Chilingarov et al. "Radiation studies and operational projections for silicon in the ATLAS inner detector ," Nuclear Instruments and Methods in Phys. Res. A, vol. 360, 1995 p. 432.

[17] M. Da Rold et al. "Radiation effects on breakdown characteristics of multi-guarded devices," IEEE Transactions on Nuclear Sciences, vol. 44, 1997 pp. 721-730.

[18] O. Militaru et al. "Study of edge effects in the breakdown process of $p^{+}$on $n$-bulk silicon diodes," Paper submitted to Elsevier Preprint, June 1998.

[19] N. Bacchetta et al. "Study of Breakdown Effects in Silicon Multi Guard Structures," 1997 IEEE Nuclear Science Symposium and Medical Imaging Conference - NSS-MIC Albuquerque,NM, USA ; 9 - 15 Nov 1997. Published in: Conference record, v.1-2 O Nalcioglu IEEE, New York, 1998, pp.498-502.
[20] L. Jones, "PREMUX specifications and user manual, version 2.3" Rutherford Appleton Laboratory internal document (1995).

[21] L. Celano et al. Nuclear Instruments and Methods in Phys. Res. A, vol. 381, 1996 p. 49.

[22] O. Adriani et al. "Beam test results for single and doublesided silicon detector prototypes of the CMS Central Detector" Nuclear Instruments and Methods in Phys. Res. A, vol. 396,1997 p. 76.

[23]' C. Bozzi "Signal-to-Noise Evaluations for the CMS Silicon Microstrip Detectors" CERN CMS Note 1997-026 (1997).

[24] U. Bondi "Caratterizzazione di dispositivi a silicio per l'esperimento CMS," Tesi di Laurea, Politecnico di Milano, Milan, Italy, October 1998.

[25] ST Microelectronics, Catania, Italy.

[26] The CMS Collaboration "The CMS Tracker Project Technical Design Report," CERN/LHCC 98-6, CMS TDR 5, 15 April 1998, Chapter 5, pp. 261-327, and references therein.

[27] S. Gadomski et al. "The deconvolution method of fast pulse shaping at hadron colliders", Nuclear Instruments and Methods in Phys. Res. A, vol. 320, 1992 p. 217. 